\title{
Creation of Computational Breast Phantoms with Extracted Abnormalities from Real Patient Images
}

\author{
Nikolay Dukov ${ }^{1}$, Zhivko Bliznakov ${ }^{1}$, Ivan Buliev ${ }^{1}$, Kristina Bliznakova ${ }^{1}$ \\ ${ }^{1}$ Technical University of Varna, Varna, Bulgaria \\ kristina.bliznakova@gmail.com
}

\begin{abstract}
The current contribution presents an approach for creation of complex computational breast phantoms, with included breast abnormalities. Inhouse developed and validated software tool, called BreastSimulator, is used to create different models of healthy breasts, by varying parameters like shape, size, duct tree features, Cooper ligaments, skin, etc. Separately, different 3D tumour models are constructed from segmented tumour regions on sets of real tomosynthesis images from real patients. A semi-automatic algorithm was developed to segment the abnormalities in the images. The algorithm applies a series of image processing operations and region-growing techniques with minimal interaction from the user to find the areas of the lesions. The flat tumour images are then correctly stacked and stored in a $3 \mathrm{D}$ voxel matrix, matching the desired resolution. Relevant information about the lesion (voxel size, matrix size, geometrical centre, etc.) is also saved. Selected tumour models are then inserted at various locations into the computational models of the healthy breasts. Examples of simulated mammographic and tomosynthesis images from such breast phantoms are presented to illustrate the potential of the proposed approach. Combining mathematical breast modelling with segmented real 3D tumour shapes results in the creation of realistic breast phantoms. The approach allows implementation of multiple scenarios and unlimited number of cases, which can be used for further software modelling and investigation of breast imaging techniques.
\end{abstract}

Keywords: breast phantom, breast simulator, tumour segmentation.

\section{$1 \quad$ Introduction}

Early diagnosis of breast cancer can significantly increase the probability for successful treatment of the disease. Therefore, many efforts are focused on improvement of the existing imaging techniques or investigations of new ones. Along that, anthropomorphic phantoms, either physical or computational, are widely used in the relevant research studies.

This work presents an approach for creation of complex computational breast phantoms, with included breast abnormalities. 


\section{$2 \quad$ Materials and Methods}

\subsection{Clinical Data}

For the current study we have used sets of tomographic images from real patients with lesions. Twenty image sets are acquired from the University Hospital of Leuven, Leuven, Belgium, using Siemens Mammomat Inspiration, while 15 image sets are acquired from Alexandrovska University Hospital, Sofia, Bulgaria, where a Giotto Tomo IMS is in service. The pixel size of the tomosynthesis images provided by the Siemens machine from the University Hospital of Leuven is 85 x $85 \mathrm{~m}$. The tomosynthesis images are obtained from 26 projection images acquired from a compressed breast. The pixel size of the tomosynthesis images provided by the Giotto machine from the Alexandrovska University Hospital is $90 \times 90 \mathrm{~m}$. The tomograms are reconstructed iteratively from 13 low-dose planar transmission images. The clinical data is priori anonymized and only technical data such as pixel values, voxel size, image dimensions, etc., are kept for the purposes of further processing and for the creation of the computational breast models and subsequent simulations.

\subsection{Breast Simulator}

For the creation of the breast models we use an in-house developed and validated software tool, called BreastSimulator (Bliznakova et al. 2010; Bliznakova et al. 2012). The tool can create models of healthy breasts, by varying parameters like shape, size, duct tree features, Cooper ligaments, skin, etc. A screenshot from the software tool is shown in Fig. 1a. The generated 3D breast phantom is then subjected to a simulation of the compression procedure applied during mammography imaging. This procedure is described in details in Zyganitidis et al (Zyganitidis et al. 2007), as the basic parameters include the desired breast compressed thickness and the elastic modulus of the breast tissues.

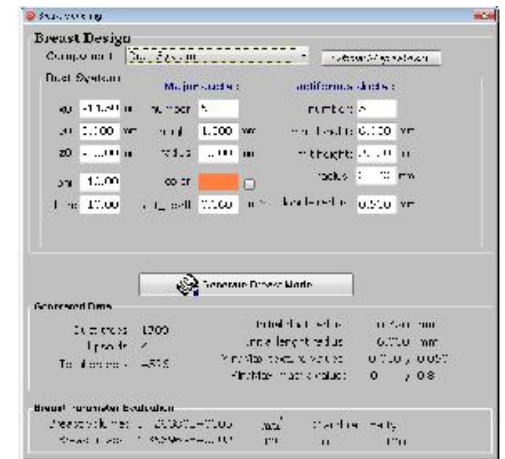

(a)

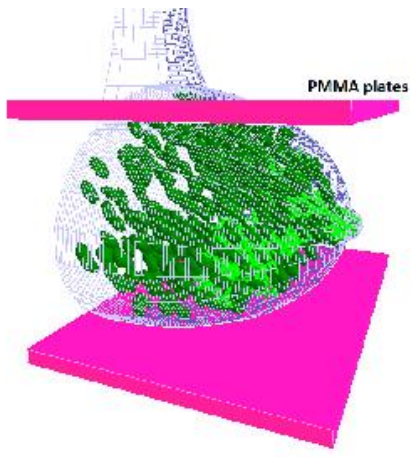

(b)

Fig. 1. A screenshot from the BreastSimulator software application, showing (a) the application user interface for modelling the duct tree and (b) the compression of a breast phantom, generated with the this tool. 


\subsection{Segmentation algorithm}

The different 3D tumour models are obtained through segmentation of tumour regions on sets of real tomosynthesis images from real patients. For the purpose, an in-house semi-automatic algorithm is used to segment the abnormalities in the images (Dukov et al. 2017; Dukov et al. 2016).

The algorithm consists of four major steps shown in Fig. 2: i) loading the clinical data, ii) pre-processing of the data, iii) segmentation of the tumour masses, and iv) creation of a 3D tumour voxel matrix.

The algorithm requires an input from the operator (user) for the initial selection the range of images at which the segmentation algorithm will be performed, as well as, a rough selection of Region of Interest (first image from the range of images). The region selection is then automatically transferred to the rest of the images.

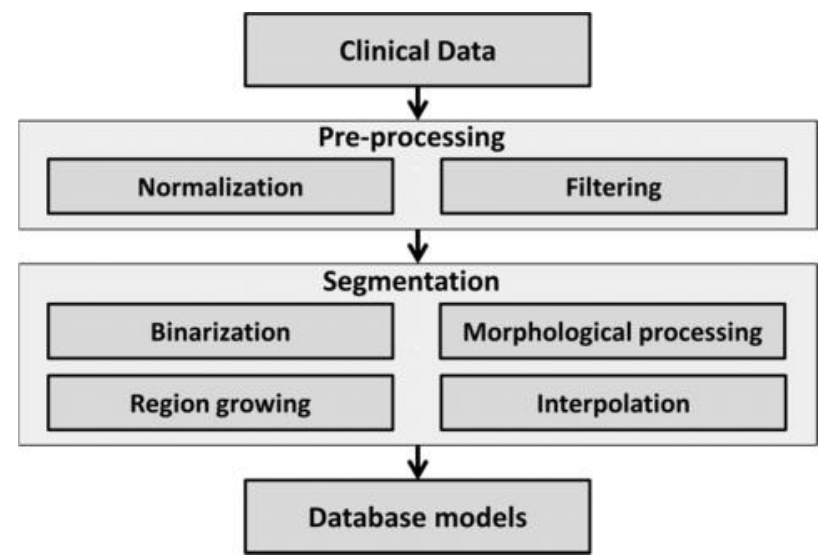

Fig. 2. Segmentation steps for the in-house developed algorithm.

Results from the different steps of the segmentation procedure are shown in Fig. 3 and present how the procedure extracts the lesion for a given slice. Finally, a 3D volume, containing the segmented shape is created and stored into a MATLAB data file.
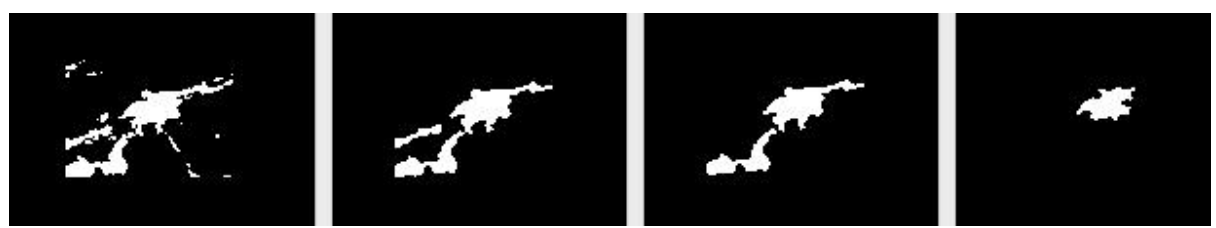

Fig. 3. Results from the different steps of the segmentation algorithm obtained after: i) thresholding; ii) morphological processing; iii) region growing; iv) interpolation.

\subsection{Insertion of extracted abnormalities into computational breast phantoms}

The results of applying the segmentation procedure on patient data are in fact computational models of breast abnormalities, represented as Boolean three-dimensional 
voxel matrices with voxel values of 1 where the abnormality is present. The user selects the place in the breast volume (phantom) where the breast abnormality will be integrated. Then the properties of the abnormality are changed in such a way that the abnormality is smoothly integrated to the surrounding tissues within the breast volume. The degree of smoothing of each voxel from the abnormality matrix depends on its distance from the centre. The more distant the voxels are, the higher degree of smoothing is achieved.

\section{$3 \quad$ Results and Discussions}

\subsection{Phantoms}

For this experimentation we generated a medium in size breast phantom with a $25 \%$ glandular tissue without skin $(33 \%$ with skin) as shown in Fig. 4a. The number of Cooper ligaments (presented as ellipsoids) is approximately 1000, while the glandular duct tree is composed of approximately 8000 cylinders of different sizes. The model is then transformed into

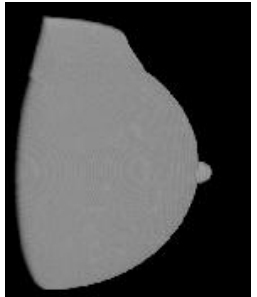

(a)

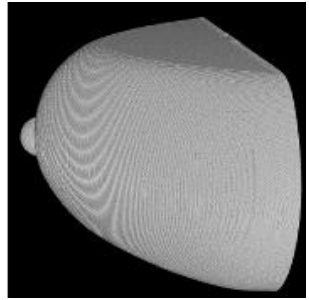

(b)

Fig. 4. Modelled breast phantom of (a) average breast $(590 \mathrm{ml})$, (b) compressed version of the computer breast model. a 3D breast voxel matrix, which contained glandular, adipose and skin tissues. Subsequently, this voxel-based breast model is subjected to a compression simulation which resulted in a breast phantom with a thickness of $4 \mathrm{~cm}$, as shown in Fig. $4 \mathrm{~b}$.

\subsection{Segmented models}

An extracted tumour model from the segmentation algorithm is shown in Fig. 5a,b.

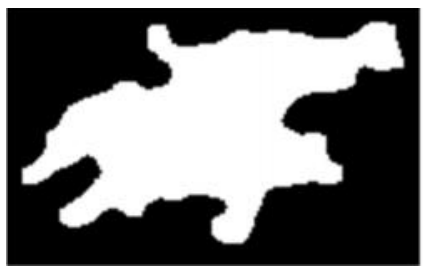

(a)

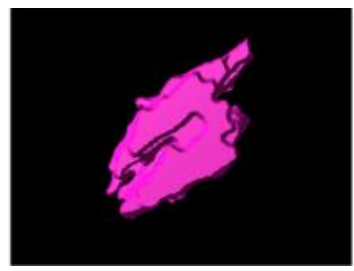

(b)

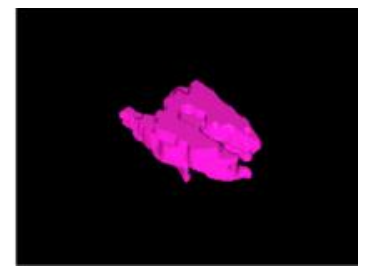

Fig. 5. An extracted model obtained from the segmentation procedure: a) $2 \mathrm{D}$ segmentation tumour area from a single slice; b) 3D visualization of the same segmented abnormality, shown from different angle views.

The tumour mass shown in Fig. 5 is segmented from 12 slices, each one with a thickness of $1 \mathrm{~mm}$. The size of the segmented tumour is $7.3 \mathrm{~mm} \times 12.3 \mathrm{~mm} \times 12 \mathrm{~mm}$. The size of the 3D matrix containing it is $96 \times 155 \times 22$ voxels. The mass was as- 
sessed subjectively by a physician (visual inspection) before introducing it into the computational breast phantom.

\subsection{Computational breast phantoms with extracted abnormalities}

\section{The 3D model}

The selected tumour model is then inserted into the computational breast phantom (Fig. 6).

\section{Simulations}

Breast tomosynthesis images with the composed breast phantom with an introduced abnormality are simulated by using XRAYImagingSimulator

(Bliznakova et al. 2010). $26 \mathrm{x}$ ray projection images of the software phantom are produced

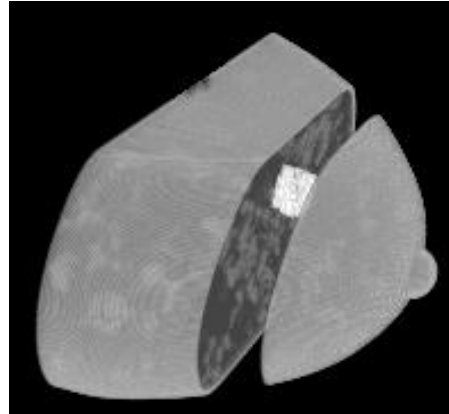

(a)

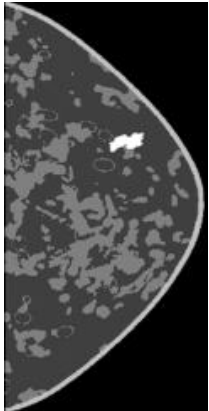

(b)

Fig. 6. Breast phantom with an introduced abnormality: a) 3D view; b) 2D view.

with energy of $20 \mathrm{keV}$ for monochromatic x-ray beam. Scatter and detector responses are not simulated. Distances from the source to the breast support table, where the phantom is placed, and to the detector surface, are $600 \mathrm{~mm}$ and $650 \mathrm{~mm}$, respectively. The size of the images is $1200 \times 1200$ pixels with pixel size of $0.085 \mathrm{~mm} \times 0.085 \mathrm{~mm}$. A mammography image obtained at craniocaudal view is shown in Fig. 7a. The 26 projection images are then used with the FDKR software application, which is an application for reconstruction of tomosynthesis slices (Bliznakova et al. 2016). Fig. 7b and Fig. 7c show the reconstructed tomosynthesis images at two different planes of the reconstructed breast phantom.

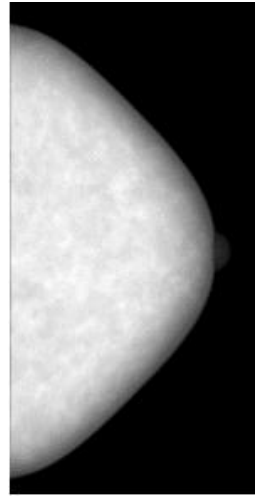

(a)

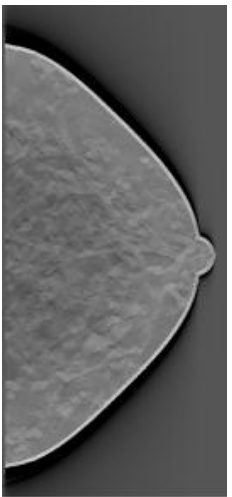

(b)

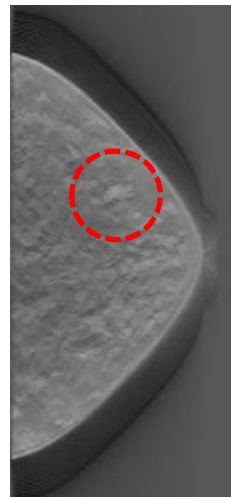

(c)

Fig. 7. Images obtained with the software phantom: (a) craniocaudal projection image, and tomosynthesis images reconstructed at two different phantom heights: (b) at the isocentric plane, (c) at the plane, where the abnormality is located. 


\section{Conclusions}

Combining mathematical breast modelling with segmented realistic 3D tumour shapes results in the creation of realistic breast phantoms, which can be used to generate realistic simulated $\mathrm{x}$-ray projection images. The approach allows implementation of multiple scenarios and unlimited number of breast tumour cases, which can be used for further software modelling and investigation of breast imaging techniques.

\section{Acknowledgments}

This work is supported by MaXIMA project, which has received funding from the European Union's Horizon 2020 research and innovation programme under grant agreement No 692097. This work is also supported by the Bulgarian National Science Fund under grant agreement DN17/2.

Conflict of interest: The authors declare that they have no conflict of interest.

\section{References}

1. Bliznakova K, et al. (2010) "Experimental validation of a radiographic simulation code using breast phantom for X-ray imaging" Computers in biology and medicine 40 208-214.

2. Bliznakova, K., P. Russo, et al. (2016). "In-line phase-contrast breast tomosynthesis: a phantom feasibility study at a synchrotron radiation facility." Phys Med Biol 61(16): 62436263.

3. Bliznakova, K., I. Sechopoulos, et al. (2012). "BreastSimulator: A software platform for breast x-ray imaging research." Journal of Biomedical Graphics and Computing 2(1): 1-14.

4. Bliznakova, K., R. Speller, et al. (2010). "Experimental validation of a radiographic simulation code using breast phantom for X-ray imaging." Comput Biol Med 40(2): 208214.

5. Bliznakova, K., S. Suryanarayanan, et al. (2010). "Evaluation of an improved algorithm for producing realistic 3D breast software phantoms: Application for mammography." Med Phys 37(11): 5604-5617.

6. Zyganitidis, C., K. Bliznakova, et al. (2007). "A novel simulation algorithm for soft tissue compression." Med Biol Eng Comput 45(7): 661-669.

7. Dukov, N., Bliznakova, K., Buliev, I., Feradov, F., Bliznakov, Z., Kostova-Lefterova, D., et al. (2017). "Development and implementation of an algorithm for segmentation of irregular lesions in Digital Breast Tomosynthesis and CT images." Book of Abstracts RAD 2017: 101. Budva.

8. Dukov, N., Feradov, F., Bliznakova, K., Encheva, E., Gluhcheva, Y., Bulyashki, D., et al. (2016). "Computational breast cancer models created from patient specific CT images: Perliminary results." Series G. Medicine, Pharmacy and Dental Medicine, Vol. IX: 106109. Plovdiv. 